\title{
Liter per Day per Milligram per Meter Squared
}

National Cancer Institute

\section{Source}

National Cancer Institute. Liter per Day per Milligram per Meter Squared. NCI Thesaurus.

Code C120731.

A unit of concentration equal to liter per day divided by milligram per meter squared. 